\title{
Modifications de la partie protidique du lait dans la stérilisation par friction
}

\author{
par \\ G. HUMBERT, D. LORIENT et Ch. ALAIS \\ Laboratoire de Biochimie Appliquée \\ Université de Nancy I - F. 54037 Nancy
}

\section{INTRODUCTION}

Le traitement thermique reste la méthode universelle pour l'assainissement du lait et l'amélioration de sa qualité de conservation ; mais, depuis le début de son application industrielle on a assisté à une évolution importante des moyens mis en œuvre, avec pour objectif principal de limiter le plus possible les modifications biochimiques et physico-chimiques résultant du mode de chauffage. Le traitement à ultra-haute température (U.H.T., $140^{\circ} \mathrm{C}-150^{\circ} \mathrm{C}$ pendant 1 à $3 \mathrm{~s}$ ) en flux continu est ainsi bien moins « traumatisant » pour le lait que la stérilisation classique en récipients hermétiques $\left(115^{\circ} \mathrm{C}-120^{\circ} \mathrm{C}\right.$ pendant 15 à $20 \mathrm{mn}$ ).

Pour aller plus loin dans cette direction, un nouveau procédé de stérilisation par friction a été élaboré. Etant donné la rapidité extrême du chauffage, ce procédé est désigné sous le terme "stérilisation Ultra-Flash » (brevet ATAD). Un programme d'études biochimiques et physico-chimiques, subventionné par la D.G.R.S.T. a été mis sur pied. L'aptitude à la coagulation a été récemment rapportée (Humbert et Alais, 1975). Dans l'étude exposée ci-après les modifications subies par la fraction protidique ont été envisagées et des comparaisons avec les procédés classiques ont été effectuées. L'état des acides aminés a été déterminé à la fois dans le lait et dans la caséine qui en provient.

\section{MATERIEL ET METHODES}

\section{Stérilisation}

Nous avons utilisé un stérilisateur pilote UF-300 ; il se compose d'un disque de $30 \mathrm{~cm}$ tournant à grande vitesse entre deux flasques fixes. L'écart très réduit entre le rotor et le stator n'est que de 3/10 
de $\mathrm{mm}$ environ; de ce fait, le lait à stériliser, entrant au voisinage du moyeu et sortant à la périphérie, subit un échauffement interne par friction extrêmement rapide.

Les paramètres temps et températures ne sont pas indépendants l'un de l'autre ; le débit est fonction de la température ; nous avons mesuré ces paramètres (tab. 1).

\section{TABLEAU 1}

Conditions de chauffage dans le stérilisateur U.F. $300^{*}$

\begin{tabular}{l|rrrrrrr}
\hline Température $\left({ }^{\circ} \mathrm{C}\right)$ & 130 & 135 & 140 & 145 & 150 & 155 & 160 \\
Durée $(\mathrm{s})$ & 0,27 & 0,41 & 0,54 & 0,68 & 0,82 & 0,96 & 1,10 \\
Débit $(\mathrm{l} / \mathrm{h})$ & 321 & 214 & 164 & 129 & 108 & 92 & 81 \\
\hline
\end{tabular}

* Température de préchauffage : $70^{\circ} \mathrm{C}$.

Des expériences comparatives ont été faites avec du lait U.H.T. commercial $\left(143^{\circ} \mathrm{C}-2 \mathrm{~s}\right)$ et du lait stérilisé à l'autoclave $\left(120^{\circ} \mathrm{C}\right.$ $15 \mathrm{mn}$ ).

\section{Répartition des matières azotées}

L'azote est dosé par microanalyse Kjeldahl. Le dosage de la fraction non caséinique (NCN) selon Rowland (1938) se fait sur le filtrat à $\mathrm{pH} 4,6$. La fraction non protéique (NPN) est dosée dans le filtrat à 12 p. 100 d'acide trichloracétique.

L'azote total du sédiment d'ultracentrifugation à $64000 \times \mathrm{g}$ pendant $30 \mathrm{mn}$ d'un volume donné de lait a été déterminé. Le culot de caséine micellaire a été dissout dans le même volume de lactosérum synthétique (Jenness et Koops, 1962) en présence de 4 p. 100 de lactose.

\section{Caséines}

Nous avons suivi la méthode de Hammarsten modifiée par Nitschman et al. (1947). Le traitement thermique par friction provoquant une certaine homogénéisation des globules gras, il a fallu écrémer les laits par ultra-centrifugation à $64000 \times$ g pendant $30 \mathrm{mn}$. Ensuite les caséines sont précipitées trois fois par $\mathrm{HCl} 1 \mathrm{~N}$, lavées à l'eau et redissoutes trois fois à $\mathrm{pH} 7$ par $\mathrm{NaOH} 1 \mathrm{~N}$, puis séchées par lyophilisation.

Malgré le champ centrifuge élevé, la séparation des lipides n'est pas totale dans le lait stérilisé à haute température. La teneur en 
substance extraite de la caséine par les solvants est de 0,34 p. 100 à $130^{\circ} \mathrm{C}, 1,5 \mathrm{p} .100$ à $140^{\circ} \mathrm{C}$ et $7,0 \mathrm{p} .100$ à $150^{\circ} \mathrm{C}$, ce qui révèle l'effet homogénéisateur du traitement.

Pour le dosage des acides aminés on a hydrolysé $1 \mathrm{mg}$ de caséine dans des tubes scellés sous vide, en présence de $0,5 \mathrm{ml}$ d'acide chlorhydrique $6 \mathrm{~N}$, à $110^{\circ} \mathrm{C}$ pendant $6,16,24$ ou $48 \mathrm{~h}$. Les acides aminés sont détectés par réaction à la ninhydrine, en gradient discontinu de $\mathrm{pH}$, à l'aide d'un autoanalyseur Technicon NC II P. La teneur de chaque acide aminé est obtenue par extrapolation au temps zéro. Une correction a été appliquée pour tenir compte de la teneur en lipides.

\section{Digestion enzymatique}

Le mélange pepsine (Worthington cristallisée) + pancréatine (Serva, no 31440 ) a été utilisé selon une technique voisine de celle de Mauron et al. (1955).

A $5 \mathrm{ml}$ d'une solution de caséine à $1,33 \mathrm{~g}$ pour $100 \mathrm{ml}$ d'eau distillée ou à 2,5 $\mathrm{ml}$ de lait additionnés de $2,5 \mathrm{ml}$ d'eau distillée, sont ajoutés $5 \mathrm{ml}$ d'une solution de pepsine (20 mg dans $100 \mathrm{ml}$ d'HCl 0,2 N).

Après $3 \mathrm{~h}$ d'incubation à $37^{\circ} \mathrm{C}$ le $\mathrm{pH}$ est élevé à 8 par addition de $5 \mathrm{ml}$ de $\mathrm{NaOH} 0,2 \mathrm{~N}$, puis sont ajoutés $5 \mathrm{ml}$ d'une solution de pancréatine $(53,33 \mathrm{mg}$ dans $100 \mathrm{ml}$ de tampon phosphate $0,05 \mathrm{M}$ à pH 8, contenant 0,04 p. 100 de merthiolate pour empêcher le développement des bactéries).

Après $24 \mathrm{~h}$ d'incubation à $37^{\circ} \mathrm{C}$ pendant lesquelles le $\mathrm{pH}$ est réajusté à 8 , la réaction est stoppée par la chaleur ( $3 \mathrm{mn}$ à $100^{\circ} \mathrm{C}$ ) et l'hydrolysat est déprotéinisé par addition d'acide trichloracétique à 12 p. 100. Après filtration et élimination du TCA par l'éther éthylique, l'hydrolysat est évaporé à sec, repris par $\mathrm{HCl} 0,1 \mathrm{~N}$ et les acides aminés libres sont dosés au moyen de l'autoanalyseur.

\section{Etude de l'état des acides aminés}

La dinitrophénylation des groupes $\mathrm{NH}_{2}$ libres a été effectuée selon Carpenter (1960), mais avec une durée de $2 \mathrm{~h}$ seulement et à $20^{\circ} \mathrm{C}$ au lieu de $30^{\circ} \mathrm{C}$. On opère avec $1 \mathrm{ml}$ d'une solution à $1 \mathrm{p} .100$ de caséine, ou $0,5 \mathrm{ml}$ de lait $+0,5 \mathrm{ml}$ d'eau distillée, auquel on ajoute $1 \mathrm{ml}$ d'une solution de FDNB (Prolabo, no 23508 ) à 2 p. 100 dans l'éthanol ; le $\mathrm{pH}$ de cette solution a été auparavant porté à 8 par addition de $\mathrm{NaHCO}_{3}$ en poudre. Après agitation pendant $2 \mathrm{~h}$ l'excès de FDNB est extrait par l'éther éthylique ; le mélange est évaporé à sec puis hydrolysé à reflux dans $\mathrm{HCl} 6 \mathrm{~N}$ pendant $24 \mathrm{~h}$ à $110^{\circ} \mathrm{C}$. Les dérivés DNP sont extraits à l'éther (méthode directe) et la phase aqueuse évaporée à sec est ensuite reprise par $\mathrm{HCl} 0,1 \mathrm{~N}$ et les acides aminés dosés par l'autoanalyseur (méthode indirecte). 
La guanidination transforme la lysine, dont le groupe $\varepsilon-\mathrm{NH}_{2}$ est libre, en homoarginine. Elle a été réalisée selon Kimmel (1967) : à $5 \mathrm{ml}$ de solution de caséine à $0,1 \mathrm{p}$. 100 ou à $3 \mathrm{ml}$ de lait $+2 \mathrm{ml}$ d'eau distillée sont ajoutés $5 \mathrm{ml}$ de réactif (O-méthylisourée- $\mathrm{HCl}$, Serva $\mathrm{n}^{\circ}$ 29380 ; $1,1 \mathrm{~g}$ pour $100 \mathrm{ml}$ d'eau distillée à $\mathrm{pH} \mathrm{10,5).} \mathrm{Après} 4 \mathrm{j}$ à $4^{\circ} \mathrm{C}$, le $\mathrm{pH}$ étant maintenu à 10,5 , la réaction est stoppée par addition d'un égal volume de tampon phosphate $1 \mathrm{M}, \mathrm{pH}$ 5. Les protéines sont ensuite isolées par dialyse et lyophilisation. La lysine non réactive et l'homoarginine apparue au cours de la réaction sont dosées, après hydrolyse acide de $24 \mathrm{~h}$ à $110^{\circ} \mathrm{C}$, par l'autoanalyseur à l'aide d'un programme d'élution des acides aminés basiques.

\section{RESULTATS}

\section{Formes de l'azote}

Le tableau 2 (moyenne de quatre expériences) montre que la variation des fractions azotées, par rapport au lait cru, est continue entre $130^{\circ} \mathrm{C}$ et $160^{\circ} \mathrm{C}$. La fraction non-caséinique (NCN) diminue de 5,8 à 10,8 par suite de la dénaturation progressive des « protéines solubles "; la fraction non-protéique (NPN) augmente de 0,3 à 0,9. La fraction sédimentable à $64000 \times \mathrm{g}$ pendant $30 \mathrm{mn}$ ne forme que 48 p. 100 de l'azote total, ce qui représente environ les $2 / 3$ de la caséine micellaire ; cette valeur augmente de 12,7 à 18,3 ; c'est-à-dire plus fortement que la caséine précipitée à $\mathrm{pH} 4,6$.

La stérilisation UHT donne des résultats correspondants à un chauffage par friction entre $150^{\circ} \mathrm{C}$ et $160^{\circ} \mathrm{C}$; or, ce dernier assure la stérilité à $140^{\circ} \mathrm{C}$; il modifie donc moins le lait. La stérilisation à l'autoclave donne les valeurs les plus élevées, correspondant à une plus grande dégradation.

\section{Variation de la composition en acides aminés de la fraction précipitée à $\mathrm{pH} 4,6$}

La fraction précipitée à $\mathrm{pH} 4,6$ du lait cru ne contient que la caséine ; sa composition en amino-acides est donnée dans le tableau 3. Après chauffage la composition de cette fraction se modifie du fait de la co-précipitation de protéines du sérum. L'action du traitement thermique peut donc être envisagée en considérant le taux d'acides aminés retrouvé dans l'hydrolysat total acide avant et après chauffage.

Le tableau 3 (détermination en double) montre que la proportion des acides aminés est modifiée après chauffage, sauf pour Glu et Met où les variations sont peu significatives. Une augmentation sensible est observée pour Thr, Ser, Gly, Ala, Val et Leu, compensée par une diminution de Pro, Ile, Tyr et Arg ; la diminution ne se produit qu'à $150^{\circ} \mathrm{C}$ pour Lys et His ; pour Asp il y a un minimum à $130^{\circ} \mathrm{C}$. 


\section{TABLEAU 2}

Variations des fractions azotées des laits chauffés à différentes températures par rapport au lait cru

\begin{tabular}{|c|c|c|c|c|c|c|c|}
\hline \multirow{2}{*}{ Azote } & \multirow{2}{*}{$\begin{array}{c}\text { Lait cru } \\
\text { (p.100 N total) }\end{array}$} & \multicolumn{4}{|c|}{ UHT-friction } & \multirow{2}{*}{$\begin{array}{c}\text { UHT } \\
143^{\circ} \mathrm{C} 2 \mathrm{~s}\end{array}$} & \multirow{2}{*}{$\begin{array}{c}\text { Autoclave } \\
120^{\circ} \mathrm{C} 15 \mathrm{mn}\end{array}$} \\
\hline & & $130^{\circ} \mathrm{C}$ & $140^{\circ} \mathrm{C}$ & $150^{\circ} \mathrm{C}$ & $160^{\circ} \mathrm{C}$ & & \\
\hline $\begin{array}{l}\text { Soluble } \\
\text { pH 4,6 }\end{array}$ & 23 & $-5,8$ & $-8,4$ & $-9,8$ & $-10,8$ & $-10,0$ & $-11,5$ \\
\hline $\begin{array}{l}\text { Soluble } \\
\text { TCA } 12 \text { p. } 100\end{array}$ & 5 & $+0,3$ & $+0,5$ & $+0,7$ & $+0,9$ & $+0,8$ & $+1,7$ \\
\hline $\begin{array}{l}\text { Sédiment } \\
64000 \times \mathrm{g}\end{array}$ & 48 & $+12,7$ & $+14,5$ & $+17,0$ & $+18,3$ & $+17,2$ & $+21,7$ \\
\hline
\end{tabular}


TABLEAU 3

Hydrolyse acide et enzymatique des caséines issues des laits chauffés ( $\mathrm{g}$ d'acides aminés pour $100 \mathrm{~g}$ de caséine)

\begin{tabular}{|c|c|c|c|c|c|c|c|c|c|c|c|c|c|}
\hline & \multicolumn{4}{|c|}{ Hydrolyse acide } & \multicolumn{8}{|c|}{ Pepsine + Pancréatine } & \multirow[b]{2}{*}{$\begin{array}{l}\text { Témoin } \\
\text { autolyse }\end{array}$} \\
\hline & $\begin{array}{l}\text { Caséine } \\
\text { témoin }\end{array}$ & $130^{\circ} \mathrm{C}$ & $140^{\circ} \mathrm{C}$ & $150^{\circ} \mathrm{C}$ & Caséin & témoin & & $\mathrm{C}$ & & $\mathrm{C}$ & & & \\
\hline ASP & 7,1 & 6,3 & 7,0 & 7,1 & & & & & & & & & - \\
\hline THR & 4,2 & 4,5 & 4,6 & 5,2 & & & & & & & & & - \\
\hline SER & 5,0 & 6,0 & 6,1 & 6,1 & 0,23 & $(4,6)$ & 0,17 & $(2,8)$ & 0,13 & $(2,1)$ & 0,13 & $(2,1)$ & 0,03 \\
\hline GLU & 21,3 & 22,1 & 21,2 & 21,4 & 0,10 & $(0,5)$ & 0,16 & $(0,7)$ & 0,13 & $(0,6)$ & 0,10 & $(0,5)$ & 0,02 \\
\hline PRO & 11,6 & 9,2 & 8,2 & 8,9 & & & & & & & & & - \\
\hline GLY & 1,8 & 2,3 & 2,4 & 2,2 & 0,27 & $(15,0)$ & 0,10 & $(4,3)$ & 0,04 & $(1,7)$ & 0,19 & $(8,6)$ & 0,02 \\
\hline ALA & 2,7 & 3,0 & 3,0 & 2,8 & 0,23 & $(8,5)$ & 0,34 & $(11,3)$ & & & 0,21 & $(7,5)$ & 0,04 \\
\hline VAL & 5,3 & 6,0 & 5,4 & 5,9 & 0,54 & $(10,2)$ & 0,51 & $(8,5)$ & 0,46 & $(8,5)$ & 0,31 & $(5,3)$ & 0,04 \\
\hline MET & 2,5 & 2,4 & 2,6 & - & 0,76 & $(30,4)$ & & & 0,10 & $(3,8)$ & 0,61 & - & - \\
\hline ILE & 5,7 & 4,6 & 4,7 & 4,8 & 0,63 & $(11,0)$ & 0,50 & $(10,9)$ & 0,63 & $(13,4)$ & 0,73 & $(15,2)$ & 0,04 \\
\hline LEU & 8,9 & 8,7 & 9,8 & 9,8 & 3,71 & $(41,7)$ & 3,07 & $(35,3)$ & 3,14 & $(32,0)$ & 3,12 & $(31,8)$ & 0,10 \\
\hline TYR & 6,3 & 5,5 & 5,1 & 5,9 & 2,15 & $(34,1)$ & 1,93 & $(35,1)$ & 1,87 & $(36,7)$ & 2,23 & $(37,8)$ & 0,08 \\
\hline PHE & 5,5 & 4,7 & 6,1 & 5,1 & 2,11 & $(38,4)$ & 1,74 & $(37,0)$ & 1,70 & $(27,9)$ & 1,98 & $(38,8)$ & 0,08 \\
\hline LYS & 8,2 & 8,4 & 8,2 & 7,7 & 3,40 & $(41,5)$ & 1,96 & $(23,3)$ & 2,40 & $(29,3)$ & 2,97 & $(38,6)$ & 0,03 \\
\hline HIS & 2,5 & 2,7 & 2,6 & 2,0 & 0,30 & $(12,0)$ & 0,24 & $(8,9)$ & 0,27 & $(10,4)$ & 0,30 & $(15,0)$ & - \\
\hline ARG & 3,4 & 3,4 & 2,7 & 2,9 & 1,63 & $(47,9)$ & 1,27 & $(37,3)$ & 1,39 & $(51,5)$ & 1,53 & $(52,8)$ & 0,04 \\
\hline
\end{tabular}


TABLEAU 4

Variation de la libération des acides aminés de laits ayant subi différents traitements thermiques (Pepsine + Pancréatine)

\begin{tabular}{|c|c|c|c|c|c|c|}
\hline & \multirow{2}{*}{$\begin{array}{l}\text { Lait cru } \\
(\mathrm{g} / 16 \mathrm{~g} \mathrm{~N})\end{array}$} & \multirow{2}{*}{$\begin{array}{c}\text { Friction } \\
140^{\circ} \mathrm{C}\end{array}$} & \multirow{2}{*}{$\begin{array}{c}\text { UHT } \\
143^{\circ} \mathrm{C} 2 \mathrm{~s}\end{array}$} & \multirow{2}{*}{$\begin{array}{c}\text { Autoclave } \\
120^{\circ} \text { C } 15 \mathrm{mn}\end{array}$} & \multicolumn{2}{|c|}{ Pasteurisations $20 \mathrm{~s}$} \\
\hline & & & & & $73^{\circ} \mathrm{C}$ & $90^{\circ} \mathrm{C}$ \\
\hline SER & 0,122 & $+0,013$ & $+0,002$ & $-0,009$ & $+0,018$ & $+0,002$ \\
\hline GLU & 0,218 & $+0,060$ & $+0,092$ & $-0,019$ & $+0,093$ & $+0,019$ \\
\hline GLY & 0,069 & $-0,005$ & $-0,016$ & $-0,012$ & $-0,009$ & $-0,007$ \\
\hline ALA & 0,252 & $+0,012$ & $+0,081$ & $+0,005$ & $+0,062$ & $+0,078$ \\
\hline VAL & 0,420 & $+0,105$ & $+0,035$ & $-0,151$ & $+0,050$ & $+0,008$ \\
\hline ILE & 0,503 & $-0,106$ & $+0,114$ & $-0,178$ & $-0,101$ & $-0,134$ \\
\hline LEU & 2,840 & $+0,520$ & $+0,420$ & $-1,106$ & $+0,377$ & $+0,281$ \\
\hline TYR & 1,613 & $-0,177$ & $-0,433$ & $-0,047$ & $+0,134$ & $+0,032$ \\
\hline PHE & 1,542 & $+0,401$ & $+0,157$ & $-0,750$ & $+0,099$ & $+0,148$ \\
\hline LYS & 2,230 & $+0,790$ & $+0,877$ & $-0,986$ & $+0,813$ & $+0,654$ \\
\hline HIS & 0,346 & $-0,220$ & $+0,170$ & $+0,046$ & $+0,135$ & $+0,149$ \\
\hline ARG & 1,483 & $+0,296$ & $+0,282$ & $-0,636$ & $+0,228$ & $+0,395$ \\
\hline
\end{tabular}




\section{Acides aminés libérés du lait par le mélange pepsine-pencréatine}

Le tableau IV (détermination en double) indique la libération des acides aminés dans le cas des laits stérilisés et pasteurisés, en prenant pour terme de comparaison le traitement par friction à $140^{\circ} \mathrm{C}$. Celui-ci permet une meilleure libération de la plupart des acides aminés que le procédé UHT commercial, mais il faut signaler quelques exceptions (Glu, Ala, Ile et Lys). La stérilisation classique à l'autoclave inhibe fortement la libération des acides aminés ; celle-ci est en effet inférieure à celle du lait cru sauf pour l'alanine et l'histidine.

La libération des acides aminés des laits pasteurisés à $73^{\circ} \mathrm{C}$ pendant $20 \mathrm{~s}$ est presque toujours supérieure (sauf pour Gly et Ile) à celle du lait cru et elle diminue en général avec l'augmentation de la température à $90^{\circ} \mathrm{C}$.

Lorsque l'on fait varier la température de stérilisation par friction on observe que la libération de tous ces acides aminés évolue de la même manière (fig. 1) : elle croît jusqu'à $150^{\circ} \mathrm{C}$ puis décroît à $160^{\circ} \mathrm{C}$, La libération est toujours plus faible à $130^{\circ} \mathrm{C}$ (sauf pour Glu) que dans le lait cru. A toute température l'acide glutamique est davantage libéré que dans le lait cru, et c'est l'inverse qui se présente pour l'isoleucine.

\section{Libération des acides aminés dans les caséines issues des laits stérilisés par friction}

Comme il a été vu dans le cas des laits correspondants, (fig. 1), la libération des acides aminés est plus faible dans les caséines des laits chauffés à faible température $\left(130^{\circ} \mathrm{C}\right)$ que dans la caséine du lait cru, sauf pour Glu et Ala ; c'est ce que montre le tableau 3 (détermination en double). Il faut remarquer que la libération de certains acides aminés diminue (Ser, Glu et Val) ou augmente (Ile, Lys, His et Arg) avec l'intensité du chauffage. D'autres acides aminés voient leur libération atteindre un maximum (Leu) ou un minimum (Gly, Tyr et Phe) à $140^{\circ} \mathrm{C}$.

Le dosage des acides aminés libérés par le mélange permet de calculer le taux d'hydrolyse enzymatique de ces caséines par rapport à leur hydrolyse acide (valeurs entre parenthèses). En moyenne environ 15 p. 100 des acides aminés totaux des caséines sont libérés en $24 \mathrm{~h}$; mais le pourcentage de libération de chaque acide aminé varie beaucoup quelle que soit la température. Ce taux est très faible dans le cas de l'acide glutamique (0,5 p. 100 environ) ; il est important dans le cas de la leucine et des acides aminés aromatiques ou basiques (autour de 50 p. 100 pour Arg, par exemple) ; il varie entre 1 et 15 p. 100 pour les autres acides aminés. 


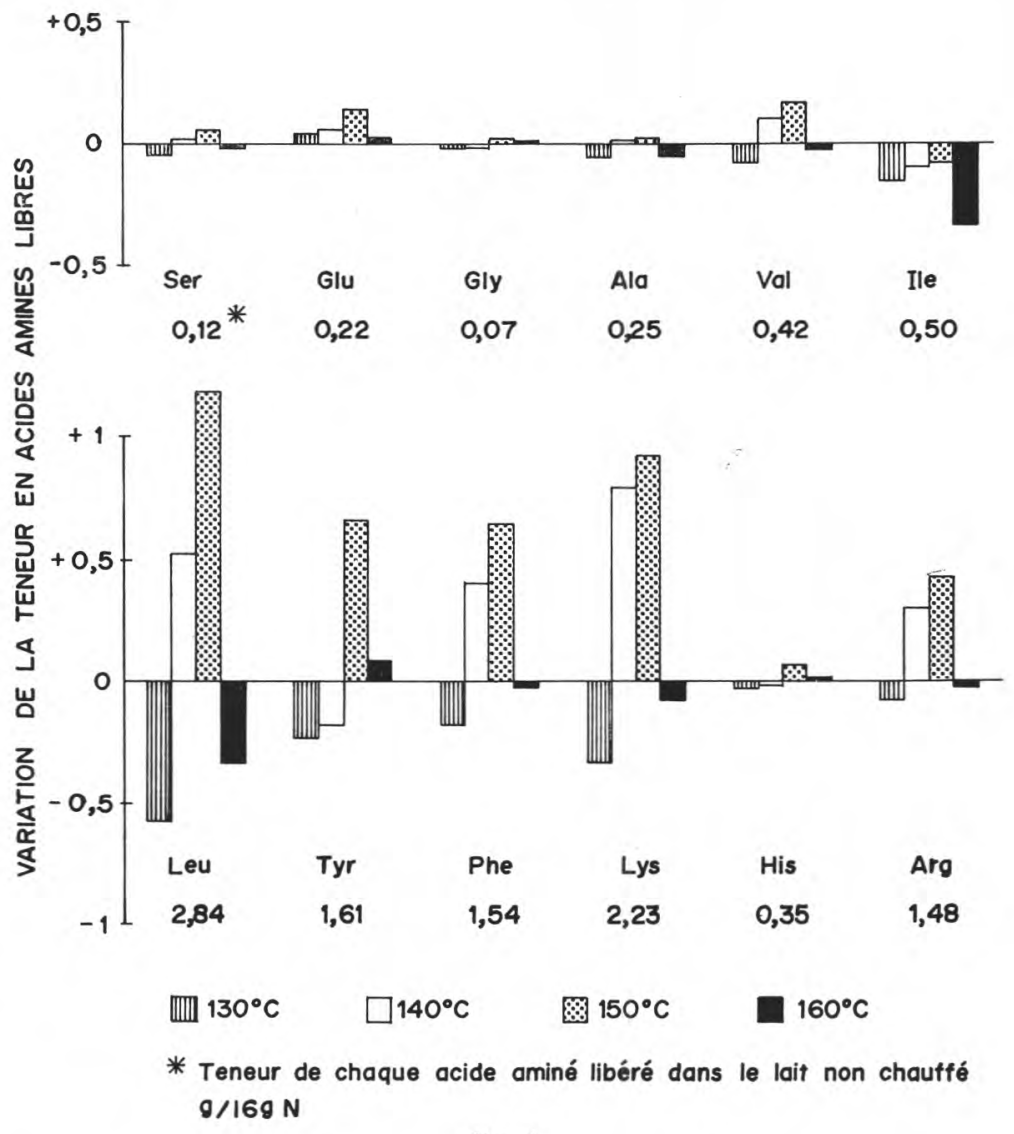

fig. 1

Variation de la libération d'acides aminés $(\mathrm{g} / 16 \mathrm{~g} \mathrm{~N})$ par le mélange enzymatique pepsine + pancréatine dans les laits chauffés par friction, par rapport au lait cru.

\section{Modifications des groupes aminés}

Le tableau 5 présente les valeurs concernant la lysine, évaluée par trois méthodes différentes, dans les laits chauffés et la caséine issue de ces laits, toutes les déterminations ayant été faites en double.

L'hydrolyse enzymatique peut révéler un blocage ou un démasquage de la lysine, par la diminution ou l'augmentation de la quantité libérée, soluble dans 12 p. 100 d'acide trichloracétique. Les effets du traitement thermique du lait, vus par ce moyen, sont variables selon les conditions. La stérilisation par friction favorise la libération de lysine à $140^{\circ} \mathrm{C}$ et à $150^{\circ} \mathrm{C}$, à peu près comme le traitement UHT 
Modifications de la lysine dans les laits chauffés et dans leur caséine (augmentation [+] ou diminution [-]) en $\mathrm{g} / 16 \mathrm{~g}$ d'azote par rapport au lait cru

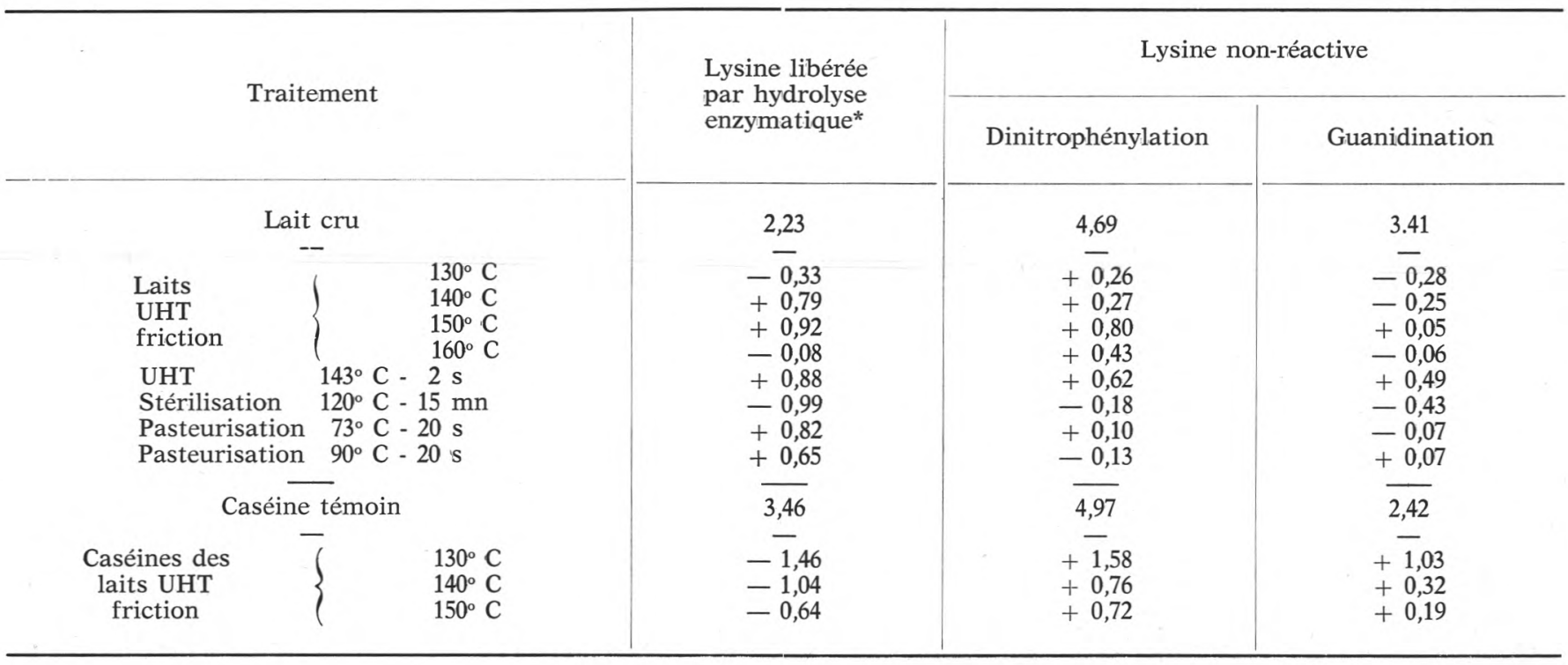

* Pepsine + Pancréatine 
commercial et la pasteurisation ; mais aux températures extrêmes, $130^{\circ} \mathrm{C}$ et $160^{\circ} \mathrm{C}$, la friction freine un peu cette libération ; on remarque que la stérilisation à l'autoclave la réduit beaucoup plus. Les résultats ne sont pas les mêmes avec la caséine provenant des laits stérilisés par friction ; une diminution sensible de la lysine libérée se produit aux trois températures appliquées ; mais elle va en s'affaiblissant avec l'intensité du traitement.

La dinitrophénylation et la guanidination permettent de déterminer la quantité de lysine non réactive. Il est à noter que la réaction du FDNB n'a pas été obtenue avec un fort rendement pour la lysine, car elle n'a pas été réalisée dans des conditions optimales, dans le dessein d'apprécier l'état de la tyrosine (Bailey, 1974 ; Lorient et al., 1975).

Les résultats obtenus par ces deux voies chimiques différentes sont comparables en ce qui concerne la caséine : à $130^{\circ} \mathrm{C}$ par friction, on observe une augmentation de la lysine bloquée, suivie d'une régression à $140^{\circ} \mathrm{C}$ et $150^{\circ} \mathrm{C}$, ce qui concorde avec les résultats de l'hydrolyse enzymatique. Dans le cas du lait stérilisé par friction, la guanidination ne révèle que de faibles changements et une tendance à la diminution de la lysine bloquée ; au contraire, une augmentation apparaît avec la dinitrophénylation, elle est maximale à $150^{\circ} \mathrm{C}$; mais il faut tenir compte du fait que la réaction a lieu en milieu complexe, la présence de sucres réducteurs pouvant provoquer une destruction partielle des dérivés DNP au cours de l'hydrolyse acide (Finot et Mauron, 1972). Les deux méthodes révèlent une augmentation dans le lait UHT commercial et peu de changement au cours de la pasteurisation. On remarque ici que la lysine est plus réactive dans le lait autoclavé que dans le lait cru ; ce résultat s'oppose donc à celui obtenu par l'hydrolyse enzymatique.

La dinitrophénylation a révélé, de plus, que les variations de la teneur en tyrosine et en arginine non réactives sont identiques à celle de la lysine au cours du chauffage.

\section{DISCUSSION}

Le nouveau procédé de stérilisation par friction (à $140^{\circ} \mathrm{C}$ $150^{\circ} \mathrm{C}$ ) dégrade moins les protéines du lait et les acides aminés que les autres procédés courants, comme il modifie moins l'aptitude à la coagulation enzymatique (Humbert et Alais, 1975). La répartition de l'azote est d'autant plus modifiée que la température est plus élevée : diminution de l'azote soluble à $\mathrm{pH}$ 4,6 (NCN) par suite de la dénaturation des protéines du lactosérum, augmentation de l'azote soluble dans l'acide trichloracétique à 12 p. 100 (NPN), augmentation de l'azote micellaire ultracentrifugeable. Il est à noter que le 
lait UHT commercial subit des modifications qui le placent à un niveau entre $150^{\circ} \mathrm{C}$ et $160^{\circ} \mathrm{C}$ par rapport au lait stérilisé par friction. Les résultats obtenus sur le lait UHT commercial sont en accord avec ceux de Samel et al. (1971), Zadow (1969) et Lyster et al. (1971). Cependant il faut noter que la forte augmentation de NPN relevée dans le lait autoclavé est beaucoup plus marquée que celle observée par Samel et al. (1971) ou Hostettler et al. (1965), alors que les variations de la fraction NCN sont très voisines. La proportion d'azote micellaire ultracentrifugeable est plus faible que celle notée par d'autres auteurs (Hostettler et al., 1965) mais il faut remarquer que les durées utilisées pour nos essais ne dépassent pas $30 \mathrm{mn}$ à $64000 \times \mathrm{g}$.

Dans nos expériences nous n'avons pas observé une baisse globale de la teneur en acides aminés de la caséine, après stérilisation du lait par friction ; en considérant chaque acide aminé, les fluctuations sont faibles. D'autres auteurs ont observé des dégradations notables d'acides aminés par chauffage de solutions de caséine isolée, dans des conditions plus sévères, par exemple, Nakanishi et Itoh (1967) qui ont noté une baisse de la teneur de la plupart des acides aminés à $120^{\circ} \mathrm{C}$ et à $140^{\circ} \mathrm{C}$ pendant $60 \mathrm{mn}$. Par contre, Bjarnason et Carpenter (1970) ont eux aussi relevé une très faible diminution des acides aminés, excepté Cys et Lys, après un long chauffage ( $27 \mathrm{~h}$ ) de la sérum-albumine bovine à $115^{\circ} \mathrm{C}$, mais en milieu peu hydraté.

La digestibilité des protéines est souvent étudiée soit in vitro en présence d'enzymes protéolytiques, soit in vivo par la croissance de rats. Selon Mauron et Mottu (1958) les deux méthodes sont nécessaires pour étudier les conséquences du chauffage des protéines. Pour Swirsky et al. (1963), De Wuyst et al. (1972), la plupart des traitements thermiques diminuent la digestibilité, à l'exception de la pasteurisation. Dans des essais comparatifs, effectués sur des laits chauffés, nous avons observé que la digestion enzymatique, mesurée par la libération des acides aminés, est améliorée sensiblement par la pasteurisation à $73^{\circ} \mathrm{C}$, et un peu moins par la pasteurisation à $90^{\circ} \mathrm{C}$ pendant $20 \mathrm{~s}$. La stérilisation par friction à $140^{\circ} \mathrm{C}$ et à $150^{\circ} \mathrm{C}$ accroît aussi la libération du plus grand nombre des acides aminés par le complexe enzymatique, plus que ne le fait le traitement UHT courant. Le traitement à l'autoclave inhibe nettement la protéolyse.

Si l'on étudie l'influence de la température dans le procédé de stérilisation par friction, on observe une inhibition de la protéolyse dans le lait à $130^{\circ} \mathrm{C}$; au contraire l'élévation de la température jusqu'à $150^{\circ} \mathrm{C}$ la favorise nettement (sauf pour l'isoleucine). Avec la caséine isolée des laits chauffés on note aussi une inhibition à $130^{\circ} \mathrm{C}$; au-dessus de cette température, l'évolution diffère suivant les acides aminés. Il est à noter que la présence des peptides acides ne permet pas une bonne séparation de l'acide aspartique, la thréonine et la proline. 
En ce qui concerne la lysine, il existe une discordance, dans le cas des laits, entre les résultats obtenus par la méthode enzymatique et par les méthodes chimiques. Les effets du chauffage ne semblent pas se traduire par une décroissance linéaire de la lysine disponible, en fonction de l'intensité du traitement thermique, mais plutôt par deux phases simultanées dont les effets se compenseraient, et dont les vitesses de réactions seraient différentes. Il s'agit d'abord d'une libération d'acides aminés, ou de l'apparition d'extrémités $\mathrm{N}$ terminales (Bjarnason et Carpenter, 1970), puis d'un blocage des groupes réactifs par le lactose (Lorient et al., 1975) ou par des groupes carboxyliques ou amidés (Mecham et Olcott, 1947). Ce double phénomène permettrait également d'expliquer que pour les caséines le blocage des groupes $\varepsilon \mathrm{NH}_{2}$ de la lysine soit moins accusé à $150^{\circ} \mathrm{C}$ qu'à $130^{\circ} \mathrm{C}$; la libération de lysine qui s'accroît par le chauffage compenserait le blocage partiel des groupes aminés à ces températures.

La lysine des protéines du lait est peu dégradée par le traitement thermique par friction à $140^{\circ} \mathrm{C}$, moins que par le procédé UHT actuel, et garde une disponibilité presque totale. On peut rapprocher cela des observations de Hill et al. $(1963,1965,1968,1970)$ selon lesquelles l'état des acides aminés basiques des caséines est en relation directe avec le phénomène de coagulation par la présure ; ceci contribue à expliquer le fait que l'aptitude à la coagulation du lait stérilisé par friction soit peu modifiée (Humbert et Alais, 1975).

\section{Remerciements}

Nous remercions la Société SEFFAC pour la mise à notre disposition du stérilisateur ATAD et nous remercions le Département de Biologie Appliquée de l'I.U.T. de Nancy pour les facilités techniques qu'il nous a offertes. Ce travail a été réalisé grâce à l'aide apportée par les contrats D.G.R.S.T. $n^{\circ}$ 73.7.1398 et 75.7.0383.

\section{Rés u mé}

Le procédé de traitement thermique par friction (procédé ATAD), appliqué dans des conditions assurant la stérilisation $\left(140^{\circ} \mathrm{C}\right)$, modifie moins la répartition des matières azotées dans le lait que la stérilisation classique et même que le traitement UHT. Les différentes méthodes enzymatiques et chimiques utilisées pour apprécier la digestibilité des protéines et l'état des acides aminés dans le lait stérilisé et dans la caséine issue de ces laits, montrent que deux phénomènes distincts se déroulent simultanément au cours du chauffage : la libération d'acides aminés et l'apparition des groupes aminés terminaux, d'une part, et les réactions de blocage d'acides aminés, d'autre part. La majeure partie de la lysine reste disponible et réactive après la stérilisation par friction. 


\section{S u $\mathrm{m} \mathbf{m}$ a $\mathbf{r} \mathrm{y}$}

The sterilization of milk by friction (ATAD process) at $140^{\circ} \mathrm{C}$ provokes relatively small changes in the nitrogen distribution by comparison with other thermic treatments. The enzymatic and chemical methods used to evaluate the protein digestibility and the state of amino acids in the sterilized milk and in casein show that two distinct phenomena are developping simultaneously during heating: first, the liberation of amino acids and the appearance of terminal amino groups and, secondly, the blocking reactions of amino acids. The greatest part of lysin remains in an available and reactive form, after friction sterilization.

Reçu pour publication en janvier 1976.

\section{Bibliographie}

Banley (C. J.) (1974). - J. Sci. Fd. Agric., 25, 1007.

BJarnason (J.) et Carpenter (K. J.) (1970). - Br. J. Nutr., 24, 313.

Carpenter (K. J.) (1960). - Biochem. J., 77, 604.

De Wuyst (A.), Vervack (W.), Charlier (H.) et Jadin (V.) (1972). - Le Lait, $517,444$.

Finot (P. A.) et Mauron (J.) (1972), - Helv. Chim. Acta, 55, 4, 1153.

Hill (R. D.) et HANSEN (R. R.) (1963). J. Dairy Res., 30, 375.

Hill (R. D.) et Laing (R. R.) (1965). - J. Dairy Res., 32, 193.

Hill (R. D.) et CRACKer (B. A.) (1968). - J. Dairy Res., 35, 13.

Hill (R. D.) (1970). - J. Dairy Res., 37, 187.

HostetrleR (H.), ImHoF (K.) et Stein (J.) (1965). - Milchwissenschaft, 20, 189.

Humbert (G.) et Alais (C.) (1975). - Revue Laitière Française, 337, 793.

Jenness (R.) et Koops (J.) (1962). - Neth. Milk Dairy J., 16, 153.

Kimmel (J. R.) (1967). - Methods in Enzymology, vol. XI, p. 584, Academic Press.

Lorient (D.), Humbert (G.) et Alais (C.) (1975). - Soumis pour publication.

Lyster (R. L. J.), Wyeth (T. C.), Perkin (A. G.) et Burton (H.) (1971). - J. Dairy Res., 38, 403.

Mauron (J.), Mottu (F.), Bujard (E.) et Egli (R. H.) (1955), - Arch. Biochem. Biophys., 59, 433.

Mauron (J.) et Motru (F.) (1958). - Arch. Biochem. Biophys., 77, 312.

Mecham (D. K.) et Olсотт (H. S.) (1947). - Ind. Engng. Chem., 39, 1023.

NaKanishi (T.) et ItoH (T.) (1967), - J. Agric. Soc. Japan, 41, 130.

Nitschman (H. S.) et Lehman (W.) (1947). - Helv. Chim. Acta, 30, 804.

Rowland (S. J.) (1938). - J. Dairy Res., 9, 42.

Samel (R.), Weaver (R. W. V.) et Gammack (D. B.) (1971). - J. Dairy Res., 38, 323.

Swirsky (M.), Allouf (R.) et Cheftel (H.) (1963). - Bull. Soc. Chim. Biol., 45, 901.

ZADow (J. C.) (1969). - The Australian Journal of Dairy Technology, 24, 44. 